Original Article (short paper)

\title{
Strength and power training improve skill performance in volleyball players
}

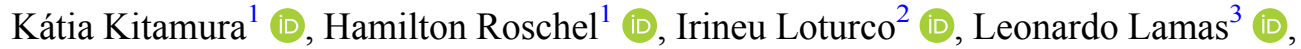 \\ Valmor Tricoli ${ }^{1}$ (D), Paulo Vicente João ${ }^{4}$ (D), Gilbert Fellingham ${ }^{5}$ (D), Carlos Ugrinowitsch ${ }^{1}$ \\ ${ }^{1}$ Universidade de São Paulo, Escola de Educação Física e Esporte, São Paulo, SP, Brasil. \\ ${ }^{2}$ Nucleo de Alto Rendimento Esportivo de São Paulo, São Paulo, SP, Brasil. \\ ${ }^{3}$ Universidade de Brasília, Brasilia, DF, Brasil. \\ ${ }^{4}$ Universidade de Trás-os-Montes e Alto Douro, Vila Real, Portugal. \\ ${ }^{5}$ Brigham Young University, Provo, United States.
}

\begin{abstract}
Aim: This study aimed to test the influence of functional improvements in volleyball skills performance. Methods: Twenty-eight amateur female volleyball players were assigned to Skill (S: $\mathrm{n}=14,19.5 \pm 4.6 \mathrm{yrs} ; 169.7 \pm$ $7.2 \mathrm{~cm} ; 62.9 \pm 12.7 \mathrm{~kg}$ ) or Skill and Strength/Power $(\mathrm{S}+\mathrm{SP}: \mathrm{n}=14 ; 18.6 \pm 3.5 \mathrm{yrs} ; 169.7 \pm 5.1 \mathrm{~cm} ; 63.3 \pm 9.2 \mathrm{~kg}) . \mathrm{S}$ + SP performed eight weeks of volleyball and conditioning training. S maintained only volleyball training. Functional variables (i.e. strength, power, and agility measures) and volleyball skills performance in a game-situation context were assessed before and after training. Results: 1-RM squat and bench press, time at modified agility T-test, peak power at bench throw and medicine-ball throw distance improved for the $\mathrm{S}+\mathrm{SP}(\mathrm{p}<0,05)$. S group increased only in medicineball throw distance $(\mathrm{p}<0.05)$. There were no differences between groups in the functional variables tested $(\mathrm{p}>0.05)$. The probabilities of a perfect set and an attack 'kill' in $\mathrm{S}+\mathrm{SP}$ improved $(\mathbb{I}>0.90)$. Changes from pre to post-training were higher in S+SP than in S for a perfect set, 'stuff block' and defense with displacement ( $\Phi>0.90)$. Conclusion: Our data suggest that functional improvements may positively affect volleyball skills performance. Although functional variables revealed only within-group changes, $\mathrm{S}+\mathrm{SP}$ produced greater improvements in skill performance than S, supporting the use of conditioning training to improve volleyball performance.
\end{abstract}

Keywords: complex training, match analyses, game-situation, athletic performance, sport performance.

\section{Introduction}

The ability of the neuromuscular system to produce power is critical to the performance in sports that require changes in direction, sprints, jumps and throws (i.e. functional abilities) $)^{1-3}$. Specifically, volleyball skills, such as serving, attacking, blocking, setting, digging and receiving the service require high levels of these functional abilities ${ }^{1-6}$.

Despite the recognized importance of improving functional abilities to sports performance, their actual contribution to enhancing skill performance is still equivocal. In this regard, a few studies have been conducted focusing primarily on the effects of physical training on sport-specific skills ${ }^{7-10}$ rather than sports performance in real match conditions.

In volleyball, conditioning coaches assume that increases in volleyball player's jumping ability, for instance, would enhance the percentage of 'kills' in the attack (i.e. attack that results in a point) and 'stuff blocks' (i.e. block that results in a point) during matches. In this point of view, it seems that functional abilities are correlated with volleyball players level, discriminating selected and non-selected young volleyball players ${ }^{11}$. Additionally, there is a significant correlation between some functional measures and game performance of each specific volleyball position (e.g. strength and digs for a defensive specialist; broad jump and block assists for middle blockers; strength and kills for outside hitters) ${ }^{12}$. Even though there is logical support for such assumptions, this may be an oversimplification of the complexity of volleyball matches. Furthermore, volleyball tactics usually require the manipulation of time and space during the rallies ${ }^{13}$. These manipulations impose faster decisional demands to the opponent and decrease the time window available to properly perform volleyball skills. For instance, if the offensive team enhances the speed of transitions from service reception to outside attacks, the defensive team would have to speed up the decision making process and the displacement of the middle blocker to form a double block, which may increase the error rates of the block ${ }^{13}$. A corollary idea is that if the defensive team can rapidly respond to the opponent's offense, the offense will be required to use faster tempo sets to overcome the defense, which may also raise attack error rates. Thus, further 
investigation into the possible effects of increasing functional abilities in volleyball skills in a game-situation context is warranted.

This study aimed to investigate if the performance of volleyball skills (i.e. service, reception, set, spike, block, and dig) is influenced by improvements in functional abilities. We hypothesized that strength and power gains would help players to better perform volleyball skills.

\section{Methods}

\section{Participants}

Twenty-eight female amateur volleyball players from two different teams volunteered to participate in the present study (Team A: $\mathrm{n}=14$ (10 attackers, 2 setters and 2 liberos); 22,6 \pm 2,8 yrs; $166,0 \pm 4,4 \mathrm{~cm} ; 64,0 \pm 13,0 \mathrm{~kg}$; $8.1 \pm 4.7$ yrs of volleyball training background; $1.35 \pm$ 0.27 relative strength in squat exercise; Team B: $n=14$ (10 attackers, 2 setters and 2 liberos); 15,6 \pm 0,5 yrs (ranged from 15 to 16 years); $173,4 \pm 5,4 \mathrm{~cm} ; 67,1 \pm 9,2 \mathrm{~kg}$; $5.1 \pm 2.2$ yrs of volleyball training background; $1.66 \pm$ 0.21 relative strength in squat exercise). Teams were engaged in volleyball practices two to five hours/day, two to four times/week, and played one to two games/week (values are referenced from A to B teams, respectively).
Each team performed technical-tactical training according to its respective coach's plan and adopted $5 \times 1$ system (5 attackers, 1 setter and 1 libero). An adherence to at least $85 \%$ of the training sessions was adopted to maintain an athlete in the study. The University's Research Ethics Committee approved the experimental protocol (CAAE: 00614112.7.0000.5391). Players from Team A and the player's parents from Team B were informed of potential risks and benefits of participation in the present study and provided their written informed consent before the commencement of the study.

\section{Measures}

Design and procedures

This was an experimental, parallel-group, and repeated-measures design. Athletes from two different volleyball teams (Teams A and B) participated in this study.

Players performed four familiarization sessions for the functional tests over two weeks. In the third week, volunteers underwent three testing sessions. During the first session, they performed the medicine-ball throw distance, half-squat, and bench press 1-RM tests. In the second testing session, countermovement jump height, time on modified agility T-test, and peak power in jump squat and bench press throw exercises were assessed. Each test-
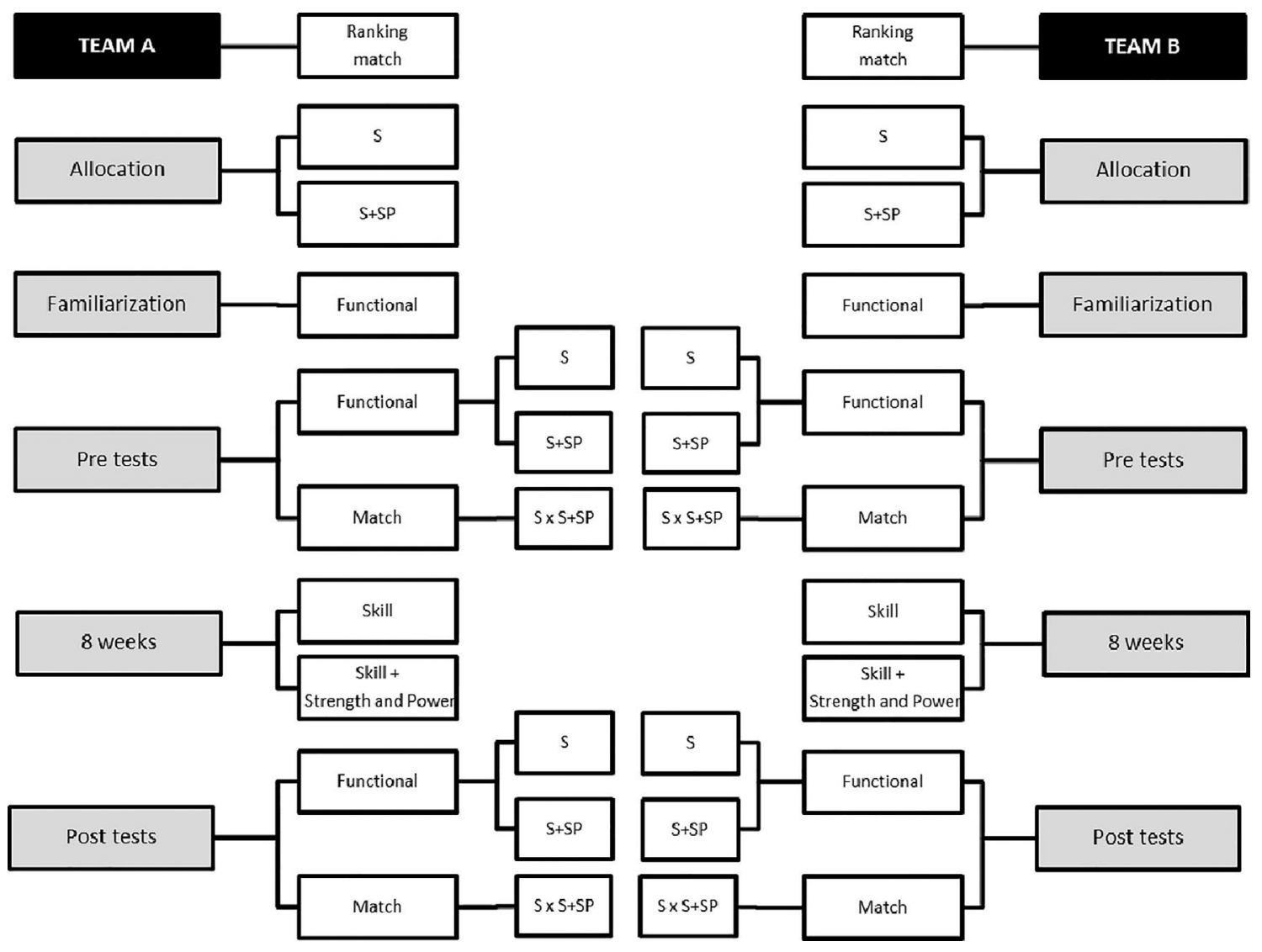

Figure 1 - Experimental design followed by both A and B teams. 
ing day started with 10 minutes of a general warm-up that consisted of running, displacements and the specific warm-up test, at a sub-maximal intensity. A volleyball exhibition match was performed in the third experimental session and a video was recorded. An interval of at least 48 hours was taken between test sessions. Both groups performed technical-tactical training for eight weeks, but only the S+SP simultaneously performed strength and power training twice a week.

Functional abilities (i.e. maximum strength, lowerand upper-limb power, and agility) and skills performance during an exhibition match (e.g. attack 'kill', 'stuff block') were assessed pre- and post-training for the same athletes (Figure 1). Importantly, each team trained separately (i.e. Team A and Team B) but followed the same conditioning protocol and experimental procedures. However, players in groups $\mathrm{S}$ and $\mathrm{S}+\mathrm{SP}$, from each team (i.e. $\mathrm{A}$ and $\mathrm{B}$ ), were collapsed for data analyses, an attempt to enhance the power of statistical tests.

\section{Pre-allocation procedures}

Players were stratified by playing positions (i.e. setters, outside hitters, opposites, middle blockers, and liberos) and classified by their respective coaches according to playing level, ranging from 1 to 4 (with 1 meaning the most skilled player and 4 the least skilled player in that specific playing position). Then, each team was divided into two sub-teams and played a four-sets ranking match, following the FIVB rules. Aiming to avoid bias, the subteam's composition was balanced according to playing level and was switched every set. Thus, middle blockers and outside hitters were allocated in pairs (i.e. 1 and 3, 2 and 4) and considered as one player for allocation purposes. This way, players had the chance to play equally twice with and against the other players (except liberos and opposites, that played always in the same sub-team, i.e., libero1 with opposite 1 and libero 2 with opposite 2). All procedures were followed equally by the two teams (A and B).

\section{Allocation procedures}

In order to allocate players into either S or S+SP, the effectiveness of each player on their specific playing position skill (i.e. reception and attack for outside hitters, attack for opposites, block and attack for middle blockers, reception and dig for liberos, and set for setters) was calculated based on the equation (1):

$$
\mathrm{E}(\%)=\frac{(\mathrm{n} 0 * 0)+(\mathrm{n} 1 * 1)+(\mathrm{n} 2 * 2)+\ldots(\mathrm{ni} * \mathrm{i})) \times 100}{\sum(\mathrm{n} 0, \mathrm{n} 1, \mathrm{n} 2 . \mathrm{ni}) * \mathrm{i}}
$$

where $\mathrm{E}=$ effectiveness, $\mathrm{n} 0$ to $\mathrm{ni}=$ frequency of actions of each result's level. 0 to $i=$ results classification of skill in accordance with its effect on opposition's actions outcome.
From the ranking match, outside hitters and middle blockers were then classified again from 1 to 4 , according to their effectiveness scores and grouped in pairs (i.e. 1 and 4; 2 and 3). Similarly, liberos and setters were classified from 1 to 2 and grouped (i.e. 1 and 2). Each pair and opposite players were allocated to either a Skill (S: $\mathrm{n}=14$, $169.7 \pm 7.2 \mathrm{~cm}, 62.9 \pm 12.7 \mathrm{~kg}, 19.5 \pm 4.6 \mathrm{yrs})$ or a Skill and strength/power group $(\mathrm{S}+\mathrm{SP}: \mathrm{n}=14,169.7 \pm 5.1 \mathrm{~cm}$, $63.3 \pm 9.2 \mathrm{~kg}, 18.6 \pm 3.5 \mathrm{yrs})$ in a random and balanced fashion, according to players' position and individual playing level, within their teams.

\section{Functional variables}

Maximal dynamic strength at squat and bench press

Maximal dynamic strength (1-RM) was tested in the half-squat (i.e. $90^{\circ}$ knee flexion) and bench press exercises (Smith Machine, Nakagym ${ }^{\circledR}$, Sao Paulo, Brazil) ${ }^{14}$. First, participants executed five minutes warm-up on a treadmill at nine $\mathrm{km} \cdot \mathrm{h}^{-1}$, followed by three minutes of lower- and upper-limb light stretching exercises. Tests were preceded by 2 warm-up sets: five repetitions set with $50 \%$ of $1-\mathrm{RM}$, and a three-repetition set with 70\% of 1-RM. Three-minute intervals were allowed between sets. Participants had up to five attempts to achieve 1-RM. Bar displacement in both half squat and bench press was controlled. Further, foot and hand positions were recorded for each exercise and reproduced in the post-training assessments ${ }^{15}$.

Time at modified agility T-test

A pair of photocells $\left(\right.$ Smart Speed ${ }^{\circledR}$, Fusion Sport, Coopers Plains, Australia) was placed at the starting/arriving line (A). From A, participants sprinted to cone B (5 $\mathrm{m})$. Facing forward and without crossing their feet, participants shuffled to the left $(2.5 \mathrm{~m})$ and then to the right (5 $\mathrm{m})$, towards cone $\mathrm{C}$ and $\mathrm{D}$, respectively. Then, they shuffled back to cone B $(2.5 \mathrm{~m})$ and sprinted backward until crossing the starting line at A $(5 \mathrm{~m})^{16}$. Each subject had three attempts and a three minutes interval was granted between attempts. The best attempt was used for further analyses.

\section{Medicine ball throw distance}

Participants threw a three kg medicine-ball as far as possible starting from the chest height. An experienced assistant researcher fixed the participants' trunk with a strap, keeping the subject's back in contact with the chair throughout the throws ${ }^{17}$. Each subject performed the throw twice with a $15 \mathrm{~s}$ interval between trials. The best trial was recorded for statistical purposes.

\section{Countermovement vertical jump height}

Participants performed a downward movement followed by a complete extension of lower-limb joints, keeping the hands on the waist. The amplitude of counter- 
movement was self-selected to avoid interferences in jumping movement pattern ${ }^{18}$. Participants performed three maximal jumps, with $15 \mathrm{~s}$ of rest interval between each trial. All of the jumps were performed on a contact resistive platform (Smart Jump ${ }^{\circledR}$, Fusion Sport, Coopers Plains, Australia) and the measured flight time ( $(\mathrm{t})$, a time interval between take-off and landing) was used to estimate the height of the rise of the body's center of mass (h) during the jumps (i.e., $\mathrm{h}=\mathrm{g} \bullet \mathrm{t}^{2} \bullet 8^{-1}$, where $\mathrm{g}=9.81 \mathrm{~m} \cdot \mathrm{s}^{-2}$ ). The highest jump height (obtained from flight time) was used for statistical purposes.

Peak power at jump squat and bench throw

Jump squat and bench throw tests were performed in a vertical Smith Machine (Technogym Equipment, Cesena, Italy). Participants were instructed to perform two repetitions with maximal speed starting with a load corresponding to $40 \%$ and $30 \%$ of their body mass in each exercise, respectively ${ }^{19}$. Jump squat was executed with participants squatted with their thighs parallel to the ground. After a signal, participants were asked to jump as high and fast as possible while maintaining the bar in contact with their shoulders. For bench throw, they were instructed to hold the bar at chest's height and throw it as high and fast as possible ${ }^{15}$. Bar load was increased by $10 \%$ body mass steps until a decrease in peak power was observed in comparison to the previous set. A 5 min rest was taken between sets. A linear potentiometer (T-Force ${ }^{\circledR}$, Dynamic Measurement System, Ergotech Consulting S.L., Murcia, Spain) was attached perpendicularly to the Smith Machine bar. Bar position data were sampled at a frequency of $1000 \mathrm{~Hz}$ and a finite differentiation technique was used to calculate bar velocity and acceleration. Peak power was obtained by multiplying force ((f), calculated as $\mathrm{f}=\mathrm{m}(\mathrm{a}+\mathrm{g})$, where $\mathrm{m}$ is the moving bar mass $(\mathrm{kg})$, and $\mathrm{a}$ is the bar acceleration and $\mathrm{g}$ is the acceleration due to gravity) by velocity during the concentric phase of the tests $^{20}$.

\section{Volleyball skills performance}

Pre- and post-training exhibition matches were video recorded from a longitudinal view and analyzed off-line. Data Volley software was used to code the actions of interest (Data Volley®, Data Project, professional version 2007). A five-sets exhibition match following International Volleyball Federation rules was performed between $\mathrm{S}$ and $\mathrm{S}+\mathrm{SP}$ groups of each Team (A and B) before and after training. The line-up of players was drawn every set in both teams and was sustained from pre- to post-exhibition matches. Players were all the same in pre- and postmatches.

Performance on the following volleyball skills that require high power production were assessed: 1) pass with displacement (i.e. a pass where a player must move to the ball); 2) perfect set (i.e. a set that gives an advantage to the attack); 3) attack 'kill' (i.e. attack point); 4) 'stuff block' (i.e. block that resulted in a point); and 5) defense with displacement (i.e. a defense where player must move to the ball). Skill execution indexes ${ }^{21}$ were assessed as binomial variables, focusing on the probability of actions produce the desired outcome (success) when considering all of the actions performed (total).

\section{Training protocol}

Training loads of the S+SP group were progressively increased throughout the eight weeks training period (Table 1). Participants performed firstly plyometrics (i.e. drop jumps and medicine ball throws) followed by resistance exercises (i.e. squat and bench press, in a free velocity, as fast as possible) involving lower and upper limbs, twice a week, with an interval of at least 48 hours between sessions. A standardized warm-up including jogging, dynamic stretching and preparatory exercises similar to training exercises but in a submaximal intensity was provided before the beginning of each training session. Plyometric training consisted of drop jumps and medicine-ball throws. During drop jumps, participants were instructed to jump as high and fast as possible. During medicine-ball exercises, participants threw the ball down and forward at chest height as fast as possible. Resistance training consisted of squat and bench press exercises in a Smith Machine (Smith Machine, Nakagym®, Sao Paulo, Brazil) and loads were traditionally and gradually increased (Table 1). Recovery intervals between plyometric exercise sets were one minute and between resistance exercises sets were three minutes.

\section{Statistical analysis}

Functional and skill execution indexes data from the players in groups $\mathrm{S}$ and $\mathrm{S}+\mathrm{SP}$, from each team, were collapsed in the following analyses. Functional variables

Table 1 - Load progression in plyometric and resistance training during 8 weeks of strength and power training.

\begin{tabular}{lccccc}
\hline Week & \multicolumn{3}{c}{ Plyometric } & & \multicolumn{2}{c}{ Strength } \\
\cline { 2 - 3 } \cline { 5 - 6 } & $\begin{array}{c}\text { Drop height } \\
(\mathrm{cm})\end{array}$ & $\begin{array}{c}\text { Medicine-ball }(\mathrm{kg}) \\
\text { weigth }(\mathrm{kg})\end{array}$ & & $\begin{array}{c}\text { Volume } \\
\left(\mathrm{S}^{\mathrm{R}}\right)\end{array}$ & $\begin{array}{c}\text { Loads } \\
\left(\mathrm{S}^{\mathrm{RM}}\right)\end{array}$ \\
\hline $\mathbf{1}$ & 30 & 1 & $5^{6}$ & $2^{10-12}$ \\
$\mathbf{2}$ & 30 & 1 & & $5^{6}$ & $3^{10-12}$ \\
$\mathbf{3}$ & 35 & 2 & & $5^{8}$ & $3^{8-10}$ \\
$\mathbf{4}$ & 35 & 2 & & $5^{8}$ & $4^{8-10}$ \\
$\mathbf{5}$ & 40 & 3 & & $6^{6}$ & $4^{8-10}$ \\
$\mathbf{6}$ & 40 & 3 & & $6^{6}$ & $3^{6-8}$ \\
$\mathbf{7}$ & 40 & 3 & & $4^{6}$ & $3^{4-6^{*}}$ \\
$\mathbf{8}$ & 40 & 3 & & $4^{6}$ & $2^{4-6^{*}}$ \\
\hline
\end{tabular}

S: number of sets; ${ }^{\mathrm{R}}$ : number of repetitions for each set; RM: repetition maximum range; * team B maintained intensity of resistance training in 68-RM in the last two weeks. 
were compared using mixed models assuming group (i.e. $\mathrm{S}$ and $\mathrm{S}+\mathrm{SP}$ ) and time (i.e. pre and post) as fixed factors and participants as a random factor. Whenever significant F values were obtained, a post-hoc Tukey's adjustment was used for multiple comparison purposes. The significance level was set at $\mathrm{P}<0.05$. Intra-class correlation coefficient and coefficient of variation of the functional variable's pre-test were $>0.90$ and $<5 \%$, respectively.

Random data samples were obtained to determine the reliability of the match analysis data. Data were obtained on two different occasions (15 days apart) and Kappa statistic was implemented to find the agreement between occasions ${ }^{22}$. Landis and Koch (1977) categories were used to determine the level of agreement for the Kappa value: $<0$ less than the chance agreement, $.01-.20$ slight agreement, $.21-.40$ fair agreement, .41 - .60 moderate agreement, $.61-.80$ substantial agreement and $.81-.99$ almost perfect agreement. All variables presented at least substantial agreement $(\geq 0.72)$. To compare the probabilities of improved skill execution indexes between the $\mathrm{S}$ and $\mathrm{S}+\mathrm{SP}$, Bayesian logistic regression models were implemented for each dependent variable using JAGS ${ }^{23}$. Posterior probabilities had to exceed 0.90 to be considered significant. Statistical software $\mathrm{R}^{\circledR 24}$ was used for all of the procedures.

\section{Results}

\section{Functional variables}

The S+SP improved 1-RM values, both in the squat $(11.2 \%, p=0.001)$ and bench press $(27.2 \%, p<0.0001)$ exercises, agility (i.e. T-test) $(6.8 \%, \mathrm{p}<0.001)$, medicineball throw $(19.1 \%, \mathrm{p}<0.0001)$, and peak power in the bench throw $(23.6 \%, \mathrm{p}<0.0001)$ from to pre- to post-training assessments (Table 2). CMJ height and jump squat peak power did not show improvements after training $(0.7 \%, p=0.98 ; 9.1 \%, p=0.14)$. On the other hand, $S$ improved only the medicine-ball throw after the experimental period $(11.0 \%, \mathrm{p}=0.0002)$. Additionally, jump squat peak power had a non-significant decrease $(-5.4 \%$, $\mathrm{p}=0.72)$ in $\mathrm{S}$. There were no significant differences between groups at the post-training period for any functional variables. The pre-post intra-group effect size ${ }^{25}$ of each functional variable was shown in Table $2^{19}$.

\section{Volleyball skills performance}

The probability of a perfect pass with displacement increased in S from pre- to post-training assessment but did not reach significance ( $(\mathbb{I}>0.81)$, while in the $\mathrm{S}+\mathrm{SP}$ it decreased over time $(\mathbb{I}>0.91)$ (Figure 2). The probability of a perfect set in S+SP was higher than in S in the pretraining, but not significantly $(\mathbb{I}>0.89)$. S showed no

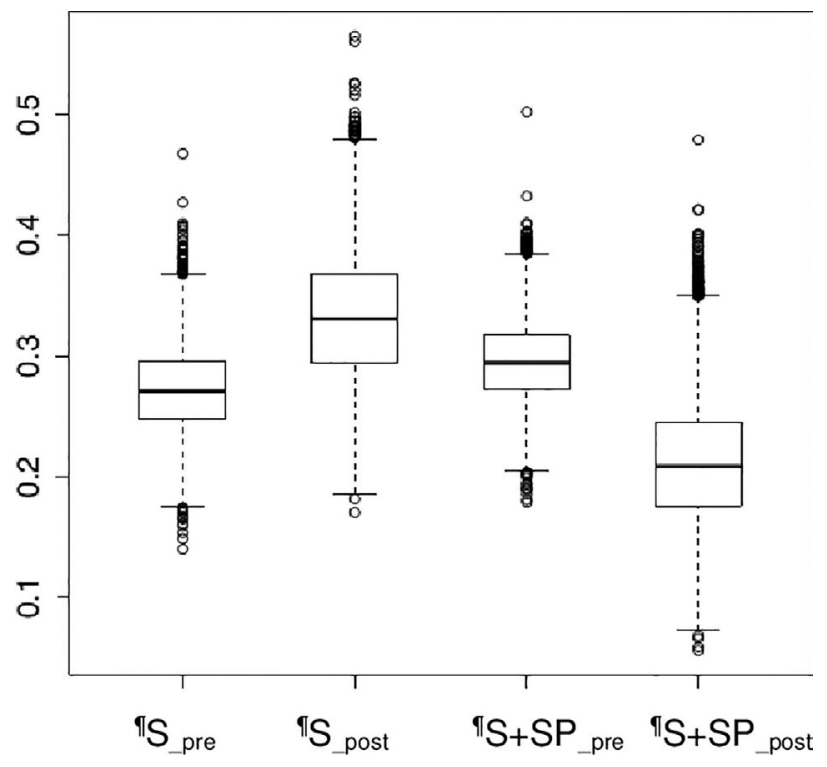

Figure 2 - Box plot of the posterior probability of volleyball perfect pass with displacement in the Skill (S) and Skill and strength/power (S+SP) groups in the pre- and post-training assessments ${ }^{36}$.

Table 2 - Comparison of functional tests results (mean $\pm S D$ ) between Skill (S) and Skill and strength/power (S+SP) groups at pre and post-training.

\begin{tabular}{|c|c|c|c|c|c|c|}
\hline \multicolumn{4}{|c|}{$\mathrm{S}$} & \multicolumn{3}{|c|}{$\mathrm{S}+\mathrm{SP}$} \\
\hline & Pre & Post & ES & Pre & Post & ES \\
\hline 1-RM_S & $96.41( \pm 22.86)$ & $96.84( \pm 26.98)$ & 0,02 & $89.29( \pm 22.74)$ & $99.30( \pm 20.00) \dagger$ & 0,44 \\
\hline 1-RM_BP & $27.19( \pm 6.27)$ & $28.78( \pm 5.92)$ & 0,25 & $25.06( \pm 8.45)$ & $31.89( \pm 8.98) \dagger$ & 0,81 \\
\hline T-test & $6.62( \pm 0.41)$ & $6.43( \pm 0.39)$ & $-0,46$ & $6.71( \pm 0.46)$ & $6.25( \pm 0.21) \dagger$ & $-0,99$ \\
\hline MBT & $309.71( \pm 37.15)$ & $346.91( \pm 41.09) \dagger$ & 1,00 & $299.42( \pm 42.53)$ & $356.57( \pm 47.96) \dagger$ & 1,34 \\
\hline $\mathrm{CMJ}$ & $28.34( \pm 3.61)$ & $27.41( \pm 3.20)$ & $-0,26$ & $28.27( \pm 4.33)$ & $28.47( \pm 3.04)$ & 0,04 \\
\hline $\mathrm{BT}_{-\mathrm{pp}}$ & $331.25( \pm 102.40)$ & $346.78( \pm 108.86)$ & 0,15 & $319.27( \pm 65.83)$ & $394.55( \pm 72.32) \dagger$ & 1,14 \\
\hline JS_pp & $967.37( \pm 193.21)$ & $915.37( \pm 189.23)$ & $-0,27$ & $835.10( \pm 189.92)$ & $911.79( \pm 201.27)$ & 0,40 \\
\hline
\end{tabular}

*S: Skill group; S+SP: Skill and strength/power group; 1-RM_s: 1-RM squat (kg); 1-RM_BP: 1-RM bench press (kg); T-test: time to agility modified Ttest (s); MBT: medicine-ball throw (cm); CMJ: countermovement jump height (cm); BT_pp: peak power in bench throw (W); JS_pp: peak power squat jump (W). $\dagger$ Intragroup significant difference from pre and post testing $(\mathrm{p} \leq 0.05)$. ES: within group effect size. No between-groups significant differences were found $(\mathrm{p}>0.05)$. 
improvement in the probability of a perfect set from preto post-training ( $\mathbb{I}>0.52)$, while $\mathrm{S}+\mathrm{SP}$ had significant improvements over time $(\mathbb{I}>0.99)$ and its change was also significantly higher than $\mathrm{S}(\mathbb{I}>0.97)$ (Figure 3 ). In S, the probability of a 'kill' increased from pre- to the post-training, but the difference was not significant ( $(\mathbb{I}>0.84)$. In $\mathrm{S}$ + SP, the probability of a 'kill' was significantly greater in the post-training period when compared with pre-training (II>0.99), but the probability that the improvement in S + SP exceeded that of the $\mathrm{S}$ was not significant $(\mathbb{I}=0.85)$ (Figure 4). The probability of a 'stuff block' decreased in $\mathrm{S}$ after training, but it did not reach significance ( $\mathbb{I}>0.88$ ). On the other hand, the probability of a 'stuff block' increased in S+SP comparing pre- to post-training, although not significantly ( $\mathbb{I}>0.84)$. The probability that the change of 'stuff block' in S+SP exceeded that of S on the post-training period was significant (II $>0.94)$ (Figure 5). The probability of a perfect dig with displacement was significantly lower from pre- to post-training in $\mathrm{S}$ (II $>0.94)$ and it was higher in S+SP, although it was not significant ( $\mathbb{I}>0.86)$. The change in $\mathrm{S}+\mathrm{SP}$ significantly exceeded the change in $\mathrm{S}$ for this variable $(\mathbb{I}>0.96)$. The probability of a perfect dig with displacement in the pretraining was slightly higher in $\mathrm{S}$ relative to $\mathrm{S}+\mathrm{SP}$, but the difference was not significant (II>0.66) (Figure 6).

\section{Discussion}

This study aimed to investigate whether increasing muscle strength and power of female volleyball players would improve performance in muscle power-dependent volleyball skills. Our main finding was that strength and

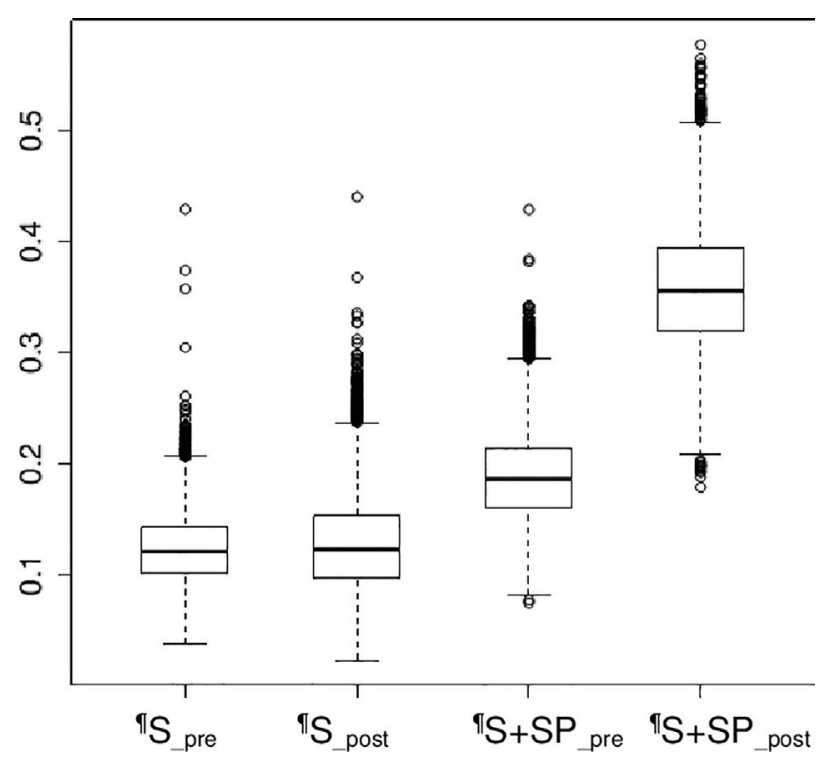

Figure 3 - Box plot of the posterior probability of volleyball perfect set in the Skill (S) and Skill and strength/power (S+SP) groups in the preand post-training assessments ${ }^{36}$.

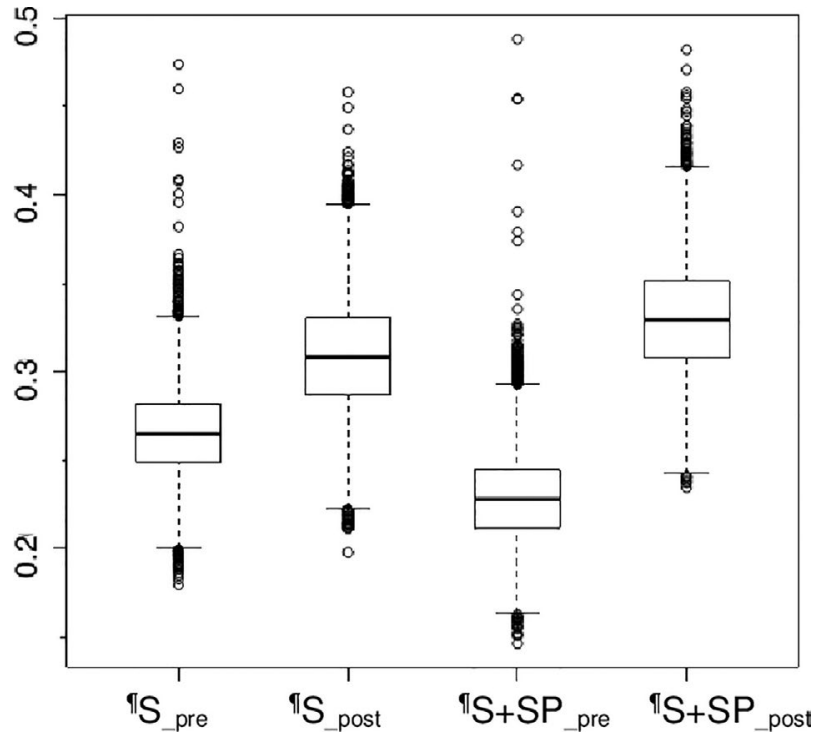

Figure 4 - Box plot of the posterior probability of volleyball 'kill' in the attack in the Skill (S) and Skill and strength/power (S+SP) groups in the pre- and post-training assessments ${ }^{36}$.

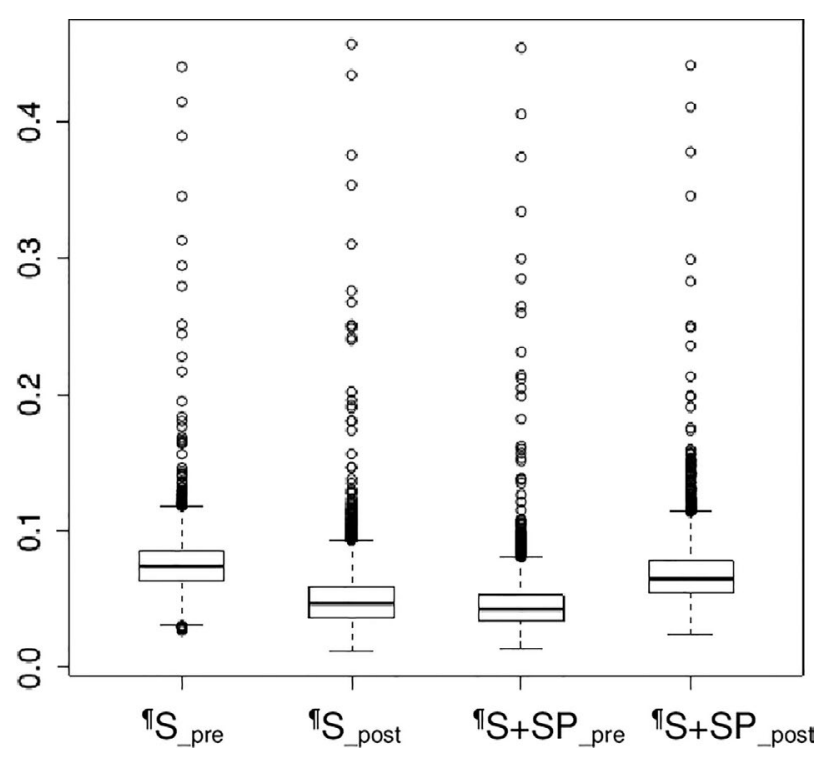

Figure 5 - Box plot of the posterior probability of volleyball 'stuff block' in the Skill (S) and Skill and strength/power (S+SP) groups in the preand post-training assessments ${ }^{36}$.

power training produced positive effects on the performance of most of the volleyball skills assessed.

The significant improvements in maximum strength, agility, and upper-limb power in S+SP are in accordance with the literature ${ }^{2,5,26-30}$ Namely, increases in squat and bench press 1-RM observed in S+SP after strength and power training are similar to those previously described $(\sim 15 \%)^{2,29,30}$. Similarly, lower- (non-significant) and upper-body peak power improvements in $\mathrm{S}+\mathrm{SP}$ are in 


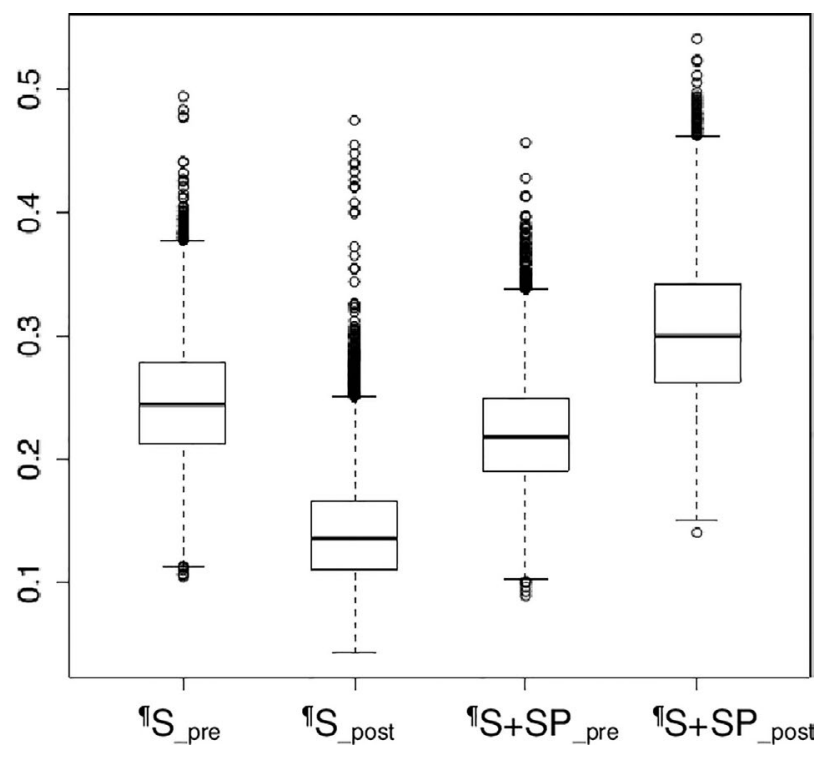

Figure 6 - Box plot of the posterior probability of volleyball perfect dig with displacement in the Skill (S) and Skill and strength/power (S+SP) groups in the pre- and post-training assessments ${ }^{36}$.

consonance to other results, after players of different sports were submitted to a period of strength and power training ${ }^{2,26}$. Strength and power training also seem to effectively increase agility and this improvement $(6.8 \%)$ is in line with the literature $(\sim 3 \%)^{27,29}$.

The test with medicine-ball improved in both groups. Indeed, some studies have shown significant improvements in this variable after strength and power training interventions ${ }^{5,30,31}$ with comparable results to ours. Although improvements in S+SP were expected, the significant gains in this variable observed in $\mathrm{S}$ may be partially explained by specific volleyball training, as the highintensity volleyball skills (e.g., attack, service, and set) may have helped developing upper body muscular power. It should be stressed that a limitation of this study is that technical/tactical training loads were not quantified properly.

While some studies have demonstrated increases in CMJ height after strength and power training ${ }^{5,30,31}$, others have found differently ${ }^{2,32,33}$. Although these findings are hard to reconcile, one may argue that, in this study, different movement patterns between training and testing situations may account for the lack of improvement. Moreover, some authors have suggested that strength gains require time to be added to the coordination pattern of jumping (lag-time) $^{2,34,35}$.

As the probability of success in performing a perfect pass increased for $\mathrm{S}$ (not significantly) and decreased for $\mathrm{S}$ $+\mathrm{SP}$, it is reasonable to speculate that the performance success of this skill may have a greater influence of technical-tactical factors (i.e. the ability of the player to get behind the ball and realize the type, trajectory, destination and velocity of the service) than by the strength and power gains. Perfect sets were influenced by the strength and power gains. S+SP presented improvements in the probability of performing a perfect set after strength and power training. Besides, changes on the probability of a perfect set after eight weeks of training were significantly higher for $\mathrm{S}+\mathrm{SP}$ when compared with $\mathrm{S}$. In this regard, gains in lower limb strength, agility and power may have benefited the setters, increasing the occurrence of more efficient techniques (i.e. jump set instead of overhand set, and overhand set instead of an underhand set) and setter ability to quickly get to the ball and adjust body position under the ball after a pass, defense or a "free ball", allowing more time to decision-making process and to properly perform the skill.

The probability of a 'kill' in the attack is related to the hitter's ability to overcome the opponent's defensive system. S+SP showed significant improvements in 'kill' probability after strength and power training. Strength and power gains seem to have helped athletes improve the chances of scoring after an attack. Improvements in bench throw power and bench press strength probably contributed to increasing ball velocity as well as attacking technique (e.g. higher occurrence of power attacks instead of tips). However, one should consider that the rate of increase in kills was not different between $\mathrm{S}$ and $\mathrm{S}+\mathrm{SP}$ groups.

Changes in the probability of a 'stuff block' were significantly higher for S+SP than S, after eight weeks of intervention. This effect was caused by the fact that $\mathrm{S}$ presented a non-significant decrease in "stuff block" and S + SP presented a non-significant increase. The number of blockers is an important factor to increase the effectiveness of blocks. Accordingly, middle blockers are trained to displace throughout the net to perform double blocks. Thus, lower-limb strength, agility and power gains may have contributed to improvements in blocking performance, suggesting that middle blockers increased the ability to perform double blocks along the entire length of the net.

Changes in the probability of a perfect dig with displacement were significantly higher for $\mathrm{S}+\mathrm{SP}$ when compared to $\mathrm{S}$ after training. Theoretically, a perfect dig with displacement could benefit from increased muscle strength and power, as players may get to the ball quicker, allowing them to perform defensive actions in a more favorable body position, thereby increasing the rate of success. This may be especially important for liberos, who are specialized in this function.

The present study has a few limitations that should be considered when analyzing our findings. First, our participants were selected from two different volleyball teams, with different coaches. Thus, the effects of distinct technical/tactical training routines due to different coaching styles could not have been properly quantified. Sec- 
ond, more exhibition methods should have been used to assess technical performance to increase the precision of the posterior probabilities estimates. Finally, our participants could not be considered as high-level volleyball players. Thus, future studies should attempt to identify the effects of strength and power training on high-level athletes.

In summary, our data suggest that eight weeks of strength and power training may positively affect volleyball skills performance.

\section{Conclusions}

Data from the present study suggest that a strength and power training program may be incorporated into specific volleyball training routines as eight weeks of conditioning strength training increased probabilities of success on some specific motor skills dependent on muscle power for volleyball athletes.

\section{References}

1. Izquierdo M, Hakkinen K, Gonzalez-Badillo JJ, Ibanez J, Gorostiaga EM. Effects of long-term training specificity on maximal strength and power of the upper and lower extremities in athletes from different sports. Eur J App Phys. 2002; 87(264-271).

2. Ronnestad BR, Kvamme NH, Sunde A, Raastad T. Shortterm effects of strength and plyometric training on sprint and jump performance in professional soccer players. $\mathrm{J}$ Strength Cond Res. 2008; 22(3): 773-80.

3. Tricoli V, Lamas L, Carnevale R, Ugrinowitsch C. Shortterm effects on lower-body functional power development: weightlifting vs. vertical jump training programs. J Strength Cond Res. 2005; 19 (2): 433-7.

4. Borras X, Balius X, Drobnic F, Galilea P. Vertical jump assessment on volleyball: a follow-up of three seasons of a high-level volleyball team. J Strength Cond Res. 2011; 25 (6): 1686-94.

5. Marques MC, Tillaar R, Vescovi JD, Gonzalez-Badillo JJ. Changes in Strength and power performance in elite senior female professional volleyball players during the in-season: a case study. J Strength Cond Res. 2008; 22(4): 1147-55.

6. Fathi A, Hammami R, Moran J, Borji R, Sahli S, Rebai H. Effect of a 16-Week Combined Strength and Plyometric Training Program Followed by a Detraining Period on Athletic Performance in Pubertal Volleyball Players. J Strength Cond Res. 2019; 33(8): 2117-27.

7. Fletcher IM, Hartwell M. Effect of an 8-week combined weights and plyometrics training program on golf drive performance. J Strength Cond Res. 2004; 18(1): 59-62.

8. Rubley MD, Haase AC, Holcomb WR, Girouard TJ, Tandy $\mathrm{RD}$. The effect of plyometric training on power and kicking distance in female adolescent soccer players. J Strength Cond Res. 2011; 25(1): 129-34.

9. Genevois C, Frican B, Creveaux T, Hautier C, Rogowski I. Effects of two training protocols on the forehand drive performance in tennis. J Strength Cond Res. 2013; 27(3): 677-82.
10. Kraemer WJ, Hakkinen K, Triplett-Mcbride NT, Fry AC, Koziris LP, Ratamess NA, et al. Physiological changes with periodized resistance training in women tennis players. Med Sci Sports Exerc. 2003; 35(1): 157-68.

11. Tsoukos A, Drikos S, Brown LE, Sotiropoulos K, Veligekas P, Bogdanis GC. Upper and Lower Body Power Are Strong Predictors for Selection of Male Junior National Volleyball Team Players. J Strength Cond Res. 2019; 33(10): 2760-7.

12. Bunn JA, Ryan GA, Button GR, Zhang S. Evaluation of strength and conditioning measures with game success in Division I collegiate volleyball: A retrospective study. J Strength Cond Res. 2017.

13. Castro J, Souza A, Mesquita I. Attack efficacy in volleyball: elite male teams. Percept Mot Skills. 2011; 113(2): 395-408.

14. Brown L, Weir J. ASEP procedures recommendation I: accurate assessment of muscular strength and power. $\mathrm{J}$ of Exe Phys2001; 4(3): 1-21.

15. Newton RU, Kraemer WJ, Kakkinen K, Humphries BJ, Murphy AJ. Kinematics, kinetics, and muscle activation during explosive upper body movements. J Appl Biomech. 1996; 12: 31-43.

16. Sassi RH, Dardouri W, Yahmed MH, Gmada N, Mahfoudhi ME, Gharbi Z. Relative and absolute reliability of a modified agility T-test and its relationship with vertical jump and straight sprint. J Strength Cond Res. 2009; 23(6): 1644-51.

17. Gillespie J, Keenum SA. A validity and reability analysis of the seated shot put as a test of power. Journal of Human Movement Studies. 1987; 13: 97-105.

18. Ugrinowitsch C, Tricoli V, Rodacki AL, Batista M, Ricard MD. Influence of training background on jumping height. J Strength Cond Res. 2007; 21(3): 848-52.

19. Dugan EL, Doyle TL, Humphries B, Hasson CJ, Newton RU. Determining the optimal load for jump squats: a review of methods and calculations. J Strength Cond Res. 2004; 18 (3): 668-74.

20. Bosco C, Belli A, Astrua M, Tihanyi J, Pozzo R, Kellis S, et al. A dynamometer for evaluation of dynamic muscle work. Eur J Appl Physiol Occup Physiol. 1995; 70(5): 379-86.

21. Memmert D HS. The Game Performance Assessment Instrument (GPAI): some concerns and solutions for further development. . J Teach Phys Educ 2008; 27: 220-40.

22. Landis JR, Koch GG. The measurement of observer agreement for categorical data. Biometrics. 1977; 33: 159-1977.

23. Plummer M. JAGS: a program for analyses of Bayesian graphical models using Gibbs sampling. Proceedings of the 3th International Workshop on Distributed Statistical Computing. 2003.

24. Team RDC. A langague and environment for statistical computing. R Foundation for Statistical Computing. 2010.

25. Rhea MR. Determining the magnitude of treatment effects in strength training research through the use of the effect size. J Strength Cond Res. 2004; 18(4): 918-20.

26. Cronin J, McNair P, Marshall RN. Velocity specificity, combination training and sport specific tasks. J of Sci Med in Sport. 2001; 4(2): 168-78.

27. Dodd DJ, Alvar BA. Analysis of acute explosive training modalities to improve lower-body power in baseball players. J Strength Cond Res. 2007; 21(4): 1177-82.

28. Fatourus, IGJ, A.Z.; Leontsini, D.; Kyriakos, T.; Aggelousis, N.; Kostopoulos, N.; Bucknmeyer, P. Evaluation of 
plyometric exercise training, weight training and their combination on vertical jumping performance and leg strength. $\mathrm{J}$ Strength Cond Res. 2000; 14(4): 470-6.

29. Harris GR, Stone HS, O’ Bryant HS, Proulx SM, Johnson RL. Short-term Performance Effects of High Power, High Force, or Combined Weight-Training Methods. J Strength Cond Res. 2000; 14: 14-20.

30. Lyttle ADW, G.J.; Ostrowski; K.J. . Enhancing performance: maximal power versus combined weights and plyometrics training. J Strength Cond Res. 1996; 10(3): 173-9.

31. Santos EJ, Janeira MA. Effects of complex training on explosive strength in adolescent male basketball players. J Strength Cond Res. 2008; 22(3): 903-9.

32. Newton RU, Rogers RA, Volek JS, Hakkinen K, Kraemer WJ. Four weeks of optimal load ballistic resistance training at the end season attenuates declining jump perfomance of women volleyball palyers. J Strength Cond Res.2006; 20 (4): 955-61.

33. Tsimahidis K, Galazoulas C, Skoufas D, Papaiakovou G, Bassa E, Patikas D, et al. The effect of sprinting after each set of heavy resistance training on the running speed and jumping performance of young basketball players. J Strength Cond Res. 2010; 24(8): 2102-8.

34. Bobbert MF, Van Soest AJ. Effects of muscle strengthening on vertical jump height: a simulation study. Med Sci Sports Exerc. 1994; 26(8): 1012-20.
35. Lamas L, Ugrinowitsch C, Rodacki A, Pereira G, Mattos $\mathrm{EC}, \mathrm{Kohn} \mathrm{AF}$, et al. Effects of strength and power training on neuromuscular adaptations and jumping movement pattern and performance. J Strength Cond Res. 2012; 26(12): 3335-44.

36. Wilke CO. Fundamentals of Data Visualization: A Primer on Making Informative and Compelling Figures. O'Reilly Media. 2019.

\section{Corresponding author}

Kátia Kitamura: Universidade de São Paulo, Escola de Educação Física e Esporte, São Paulo, Brasil. Av. Prof. Melo Moraes 65, São Paulo, Brasil.

E-mail: katiapascoto@yahoo.com.br.

Manuscript received on February 10, 2019

Manuscript accepted on November 22, 2019

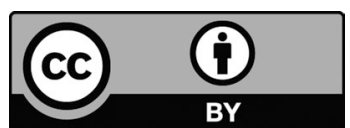

Motriz. The Journal of Physical Education. UNESP. Rio Claro, SP, Brazil - eISSN: 1980-6574 - under a license Creative Commons - Version 4.0 